\title{
Evaluation of the of antibacterial efficacy of polyvinylpyrrolidone (PVP) and tri-sodium citrate (TSC) silver nanoparticles
}

\author{
Arindam Dey $^{1,2}$ - Abhirup Dasgupta ${ }^{1,2} \cdot$ Vijay Kumar $^{1} \cdot$ Aakriti Tyagi $^{1}$. \\ Anita Kamra Verma ${ }^{1}$
}

Received: 6 April 2015/Accepted: 21 August 2015/Published online: 16 September 2015

(C) The Author(s) 2015. This article is published with open access at Springerlink.com

\begin{abstract}
We present silver nanoparticles as the new age broad spectrum antibiotic. Siver nanoparticles exhibit unique physical and chemical properties that make them suitable for understanding their biological potential as antimicrobials. In this study, we explored the antibacterial activity of silver nanoparticles (TSC-AgNPs) and silver nanoparticles doped with polyvinylpyrrolidone (PVPAgNPs) against Gram-negative and Gram-positive bacteria, Escherichia coli (DH5 $\alpha$ ) and Staphylococcus aureus, (ATCC 13709). Nucleation and growth kinetics during the synthesis process of AgNPs were precisely controlled using citrate (TSC) and further doped with polyvinylpyrrolidone (PVP). This resulted in the formation of two different sized nanoparticles 34 and $54 \mathrm{~nm}$ with PDI of 0.426 and 0.643 . The physical characterization was done by nanoparticle tracking analysis and scanning electron microscopy, the results of which are in unison with the digital light scattering data. We found the bactericidal effect for both TSCAgNPs and PVP-AgNPs to be dose-dependent as determined by the minimum inhibitory concentration (MIC) and minimum bactericidal concentration (MBC) against $E$. coli and $S$. aureus. Interestingly, we also observed that AgNPs showed enhanced antimicrobial activity with a MIC of 26.75 and $13.48 \mu \mathrm{g} / \mathrm{ml}$ for E. coli and S. aureus, respectively, while MBC for AgNPs are 53.23 and $26.75 \mu \mathrm{g} / \mathrm{ml}$ for E. coli and S. aureus, respectively. Moreover, AgNPs
\end{abstract}

A. Dey and A. Dasgupta have contributed equally.

Anita Kamra Verma

akamra23@hotmail.com; akverma@kmc.du.ac.in

1 Nanobiotech Lab, Department of Zoology, K.M. College, University of Delhi, Delhi 110 007, India

2 School of Biotechnology, KIIT, Bhubaneshwar, Odisha, India showed increased DNA degradation as observed confirming its higher efficacy as antibacterial agent than the PVP doped AgNPs.

Keywords Silver nanoparticles · Citrate .

Polyvinylpyrrolidone $\cdot$ MIC $\cdot$ MBC

\section{Introduction}

The field of nanoscience is one of the most active areas of research over the last 20 years. Nanotechnology is important as miniaturization apparently improvises applications in areas such as computing, sensors, and biomedical research [1-3]. Nanoparticles exhibit completely new or improved properties owing to their specific characteristics, such as size, distribution, and morphology and therefore used in a wide range of potential applications such as medicine, cosmetics, renewable energies, environmental remediation and biomedical devices. Silver nanoparticles (AgNPs or nanosilver) have attracted increasing interest due to their unique physical, chemical and biological properties compared to their macro-scaled counterparts [4]. AgNPs have distinctive physico-chemical properties, including a high electrical and thermal conductivity, surface-enhanced Raman scattering, chemical stability, catalytic activity and nonlinear optical behavior [5]. These properties make them of potential value in inks, microelectronics, and medical imaging [6]. And also used in a diverse range of consumer products, including plastics, soaps, pastes, food and textiles, increasing their market value [7-9]. Nanosilver can be used in a liquid form, such as a colloid (coating and spray) or contained within a shampoo (liquid) and can also appear embedded in a solid such as a polymer master batch or be suspended in a bar of soap (solid). Nanosilver (AgNPs) is 
reported to possess anti-fungal [10], anti-inflammatory [11], and anti-viral activity [12].

In recent years, resistance to antibiotics by pathogenic bacteria and fungi has been increasing at an alarming rate and has become a serious problem. For example, antibioticresistant forms of pathogenic S. aureus (e.g. MRSA) is a worldwide problem in clinical medicine [13] and also some strains of $E$. coli $(\mathrm{O} 157: \mathrm{H} 7)$ show resistance to a variety of antibiotics such as ampicillin, kanamycin, sulfisoxazole, streptomycin, tetracycline, ticarcillin, and so forth [14]. Silver has been thought of as a promising agent for overcoming the resistance mechanism of antibacterial action on a range of targets as compared to a specific site of action in the case of antibiotics. The synergistic activity of AgNPs coupled with antibiotics has been reported earlier [15]. Hence, silver-based nanoparticles could be effective bactericidal materials as they will exhibit combined effects of silver and antibacterial agents. In the present study, we have compared the effects of two different stabilizing agents i.e. citrate and polyvinyl pyrrolidone used in the synthesis of AgNPs.

\section{Materials and methods}

Nutrient broth, agarose, nutrient agar and gentamycin were purchased from Hi-Media Laboratories Pvt. Ltd. (Mumbai, India). Glucose, sodium chloride and isopropanol were purchased from CDH Lab New Delhi, India. Bromophenol blue dye and EDTA were purchased from Sisco-Research Lab Pvt Ltd. Tris buffer was purchased from Qualigens fine chemicals (Mumbai, India and), Sodium Dodecyl Sulphate (SDS) was purchased from Bio Rad Laboratories, sodium hydroxide was purchased from E-Merck limited. Silver nitrate $\left(\mathrm{AgNO}_{3}, 99.99 \%\right)$, tri-sodium citrate dihydrate $\left(\mathrm{C}_{6} \mathrm{H}_{5} \mathrm{O}_{7} \mathrm{Na}_{3} \cdot 2 \mathrm{H}_{2} \mathrm{O}, \quad 99.99 \%\right)$, sodium borohydride $\left(\mathrm{NaBH}_{4}, \quad 98 \%\right), \quad$ polyvinylpyrrolidone (PVP; $\left.M_{\mathrm{w}}=40,000\right)$ were purchased from Merck, USA. $E$. coli, DH5 $\alpha$, was procured from the repository at PGI Chandigarh. Deionized water was used throughout the experiment.

\section{Preparation of silver nanostructures}

AgNPs were prepared according to a previously published protocol by Fang et al. [16] by reduction of aqueous $\mathrm{AgNO}_{3}$ with sodium citrate at boiling temperature. Briefly, $50 \mathrm{ml}$ of $0.001 \mathrm{M} \mathrm{AgNO}_{3}$ was heated to $90{ }^{\circ} \mathrm{C}$. To this solution, $5 \mathrm{ml}$ of $1 \%$ tri-sodium citrate was added drop wise and the temperature was maintained under continuous stirring. The reaction was allowed to take place until the color changed to a pale yellow solution, which was then cooled to room temperature. The mechanism of reaction that could be expressed to prepare AgNPs is as follows:
$4 \mathrm{Ag}^{+}+\mathrm{C}_{6} \mathrm{H}_{5} \mathrm{O}_{7} \mathrm{Na}_{3}+2 \mathrm{H}_{2} \mathrm{O}$

$\rightarrow 4 \mathrm{Ag}^{\mathrm{o}}+\mathrm{C}_{6} \mathrm{H}_{5} \mathrm{O}_{7} \mathrm{H}_{3}+3 \mathrm{Na}^{+}+\mathrm{H}^{+}+\mathrm{O}_{2} \uparrow$

The synthesized AgNPs were doped using $\mathrm{NaBH}_{4}$ and PVP as reducing and stabilizing agent, respectively. An aqueous solution of tri-sodium citrate $(0.5 \mathrm{ml}, 30 \mathrm{mM})$ was added into a flask (50 $\mathrm{ml}$ deionized water), and further mixed with aqueous solution of $\mathrm{AgNO}_{3}(1 \mathrm{ml}, 5 \mathrm{mM})$. Freshly prepared $\mathrm{NaBH}_{4}$ aqueous solution $(0.5 \mathrm{ml}$, $50 \mathrm{mM}$ ) was immediately added that gave a light yellow color solution. In this solution, PVP $(0.5 \mathrm{ml}, 5 \mathrm{mg} / \mathrm{ml}$, $\left.M_{\mathrm{w}}=40,000\right)$ was added after $30 \mathrm{~s}$ at room temperature. These PVP doped silver nanoparticles were marked PVPAgNPs.

\section{Characterization of silver nanoparticles}

\section{$U V-$ Vis spectroscopy}

The appearance of yellow color in the reaction mixtures was observed within minutes, an obvious indication for the silver nanoparticle formation. Furthermore, the nanoparticle synthesis was assured by monitoring the absorption spectra in Cary $60 \mathrm{UV}-\mathrm{Vis}$ spectrophotometer, of the synthesized colloidal solutions, against distilled water using as blanks.

\section{Dynamic light scattering}

The sizes of AgNPs were observed by Zetasizer (Nano-ZS, Malvern USA) [17]. Briefly, the sample solution was diluted with deionized water to isolate all individual nanoparticles from the aggregates to assess the distribution based on size and surface charge of nanoparticles. The migration voltage was fixed at $100 \mathrm{mV}$ for all measurements. The instrument was calibrated against $10^{-4} \mathrm{M} \mathrm{AgI}$ colloidal dispersions. To measure the mean value of nanoparticles core radius, Ultraviolet-Visible spectrophotometry was done.

\section{Nanoparticle tracking analysis (NTA)}

Nanoparticle tracking analysis measurements were done with a NanoSight NS 300 (NanoSight, Malvern, United Kingdom), equipped with a sample chamber with a 640-nm laser and a Viton fluoro-elastomer O-ring [18]. Using sterile syringes, requisite amount of samples was injected in the sample chamber till the liquid reached the tip of the nozzle. All measurements were performed at room temperature. The software used for capturing and analyzing the data was the NTA 2.0 Build 127 . The samples were measured for $40 \mathrm{~s}$ with manual shutter and gain adjustments. 


\section{Scanning electron microscopy}

Scanning electron microscopy (SEM) was done to characterize the morphology of the silver nanoparticles. The silver nanoparticles were viewed with an EVO LS 10 (Carl Zeiss, Brighton, Germany) scanning electron microscope operating at an accelerating voltage of $13.52 \mathrm{kV}$ under high vacuum. Data analysis was done using Smart SEM software program [19].

\section{Antibacterial efficacy}

\section{Preparation of bacterial culture media}

Appropriate amount of nutrient broth was dissolved according to manufacturer's instruction. Broth was autoclaved at $121^{\circ} \mathrm{C}, 15 \mathrm{psi}$ pressure for $20 \mathrm{~min}$. Mother inoculum of bacterial cultures was taken in 1:100 ratios and added to the above sterile nutrient broth. The flask was incubated overnight at $37{ }^{\circ} \mathrm{C}$ in the orbital shaker incubator. Next day turbidity of media indicated the growth of the culture. Absorbance was measured at a $600 \mathrm{~nm}$ by $\mathrm{UV}$ spectrophotometer and $\mathrm{CFU} / \mathrm{ml}$ was calculated as a ratio between the optical density and $\mathrm{CFU} / \mathrm{ml}$, $1 \mathrm{OD}=0.8 \times 10^{9} \mathrm{CFU} / \mathrm{ml}$.

\section{Disc diffusion assay and analysis of growth curve}

All the glassware and media used were sterilized in an autoclave at $121{ }^{\circ} \mathrm{C}$ for $20 \mathrm{~min}$. The bacterial strains E. coli $(\mathrm{DH} 5 \alpha)$ and $S$. aureus were used as model test strains for Gram-negative and Gram-positive bacteria. The bacterial suspension was prepared in nutrient broth at $37^{\circ} \mathrm{C}$ overnight. The antibacterial susceptibility of AgNPs was evaluated using the Kirby Bauer method. Bacterial suspension was mixed with soft agar and poured on a LB agar plate. The plates were then divided into sections and sterile discs of $5 \mathrm{~mm}$ diameter were placed on each section loaded with $20 \mu \mathrm{l}$ of AgNPs [TSC-AgNPs $(107.86 \mu \mathrm{g} / \mathrm{ml})$ and PVP-AgNPs $(539.43 \mu \mathrm{g} / \mathrm{ml})]$, Gentamycin (positive control) and Distilled water (negative control); the plates were further incubated overnight at $37{ }^{\circ} \mathrm{C}$. The zone of inhibition (ZoI) was measured after $24 \mathrm{~h}$.

Bacterial cultures were inoculated to the freshly prepared nutrient broth by adding stock culture to Nutrient broth in 1:100 ratios and kept in $37{ }^{\circ} \mathrm{C}$ incubator for $24 \mathrm{~h}$. The different samples were prepared by adding the inoculated culture $(100 \mu \mathrm{l})$ to the equally divided media, control (flask containing nutrient media, with only inoculum and no AgNPs) and experimental flasks (flask containing inoculum and nutrient media, with AgNPs). The absorbance values for experimental flasks (containing nutrient media, inoculum and AgNPs) were corrected by deducting the corresponding absorbance values for the control. Here, antibacterial effects of AgNPs were studied using optical intensity as function of time for $25 \mathrm{~h}$ for different size of AgNPs.

\section{Determination of MIC and MBC}

To examine the minimum inhibitory concentration (MIC), and minimum bactericidal concentration (MBC) of different sized AgNPs, the procedure specified by Ruparelia et al. [20] was followed. The effect of AgNPs on bacterial growth kinetics was measured at $600 \mathrm{~nm}$, as increase in absorbance using a spectrophotometer. The experiment included a positive control (flask containing AgNPs and nutrient media, without inoculum) and a negative control (flask containing inoculum and nutrient media, without AgNPs). The absorbance values for experimental flasks (containing nutrient media, inoculum and AgNPs) were corrected by deducting the corresponding absorbance values for the positive controls. In this experiment we have taken three concentrations of AgNPs, 13, 26.75, $53 \mu \mathrm{g} / \mathrm{ml}$ and in case of PVP-AgNPs three concentrations are 67.3, 134.75 , and $269.5 \mu \mathrm{g} / \mathrm{ml}$.

\section{DNA fragmentation assay}

DNA damage study was performed using our previous published protocol [21] on gram-negative bacteria, E. coli and gram-positive bacteria $S$. aureus, after requisite treatment with the prepared AgNPs. Briefly, the cells were pelleted and resuspended in Glucose-Tris-EDTA buffer at $\mathrm{pH}$ 8. Cells were lysed by addition of $1 \%$ SDS. After dissolving the lipid component of the cell envelope, $\mathrm{NaCl}$ was added. Further, the cells were treated with phenol:chloroform:isoamyl alcohol in the ratio of 25:24:1 that helped in protein precipitation. The precipitate was collected after discarding the supernatant. Isopropanol was then added. To further remove the remaining salts and SDS, the precipitate was washed with ethanol, and the pellet was left overnight to dry. DNA was resolved and electrophoresed in $1.5 \%$ agarose gel at $60 \mathrm{~V}$. The gel was stained with ethidium bromide and the fragments of DNA were observed in UV light.

\section{Results}

\section{Characterization of silver nanoparticles}

\section{$U V-$ Vis spectroscopy}

In the UV-Vis spectra (Fig. 1), a single strong peak was observed for citrate and PVP capped AgNPs at 415 and 
Fig. 1 UV-Vis spectra of citrate and PVP doped AgNPs

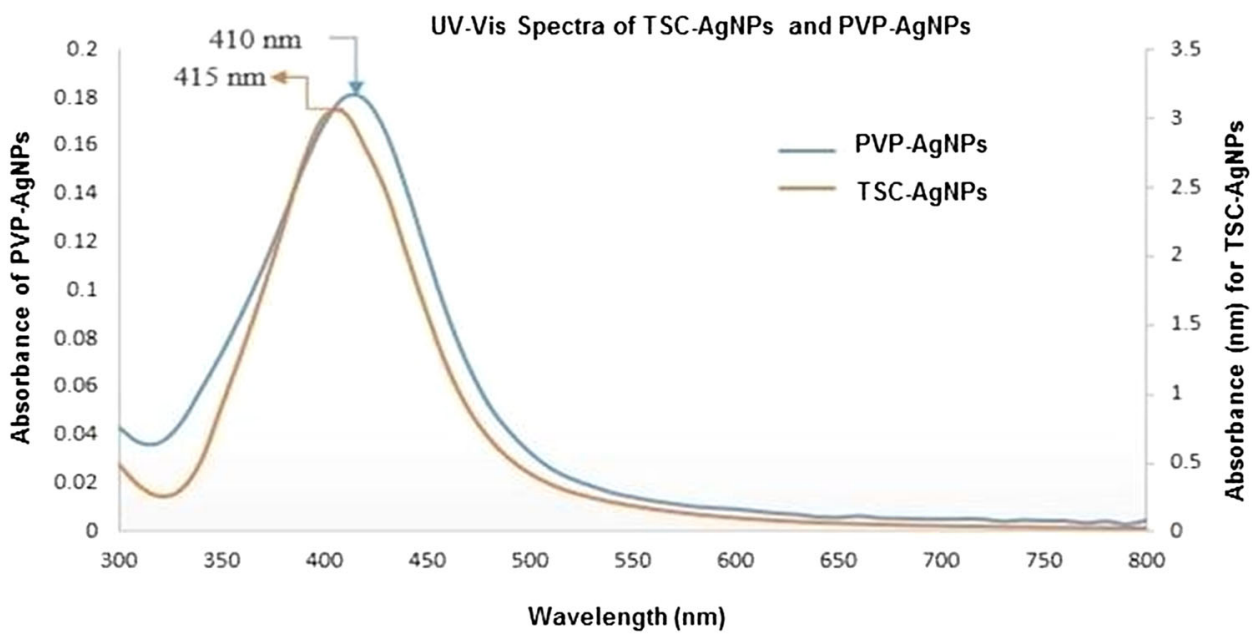

A

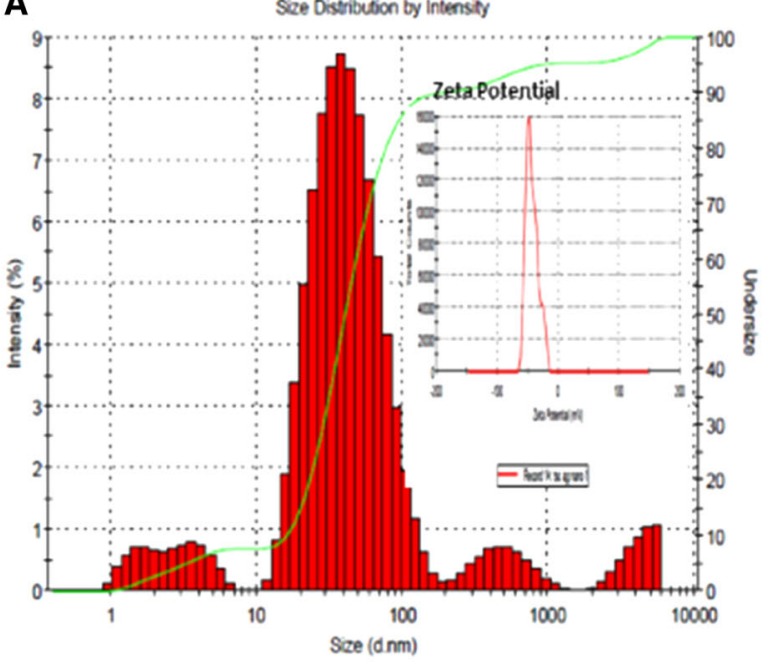

Intensiny (Record 9. AgNPS (TSC) 1)

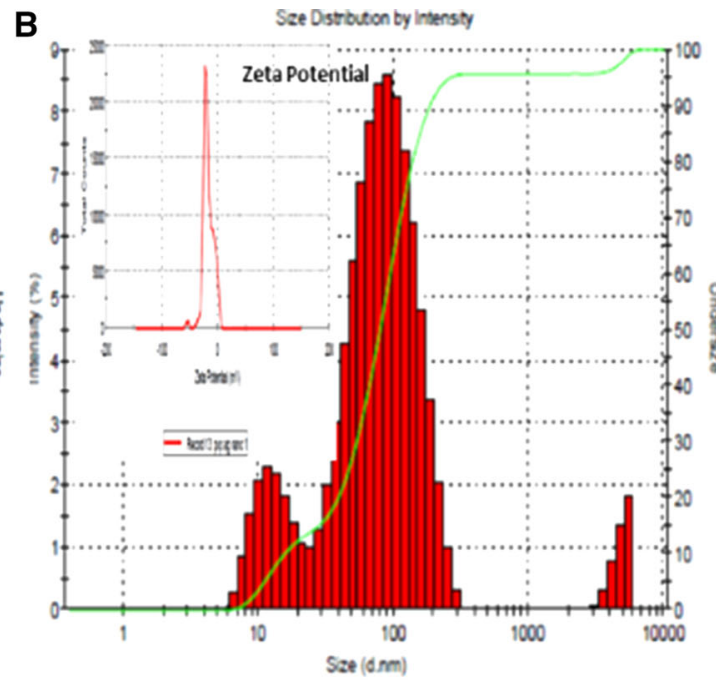

Intensity Record 8: AgNPs (PVP) 1) —Undersize (Recond 8. AgNPs (PVP) 1]

Fig. 2 DLS measurements showing a TSC- AgNPs mean diameter $(34.01 \mathrm{~nm})$ with polydispersity index 0.426 and $\zeta=-42.9 \mathrm{mV}$. b PVPAgNPs mean diameter $(54.09 \mathrm{~nm})$ with polydispersity index 0.643 and $\zeta=-16.9 \mathrm{mV}$

$410 \mathrm{~nm}$, respectively, which corresponds to the typical surface plasmon resonance (SPR) of spherical AgNPs. When compared to TSC, the SPR of PVP stabilized nanoparticles has shown blue shift towards a shorter wavelength of $410 \mathrm{~nm}$. The shift in SPR is determined by the capping agent due to the local nature of its effect on the surface of the nanoparticles.

\section{Dynamic light scattering}

Dynamic light scattering was used to determine the size distribution profile of nanoparticles in suspension. AgNPs synthesized using TSC as a reducing and stabilizing agent showed an average size of $34.01 \mathrm{~nm}$ with $0.42 \mathrm{PDI}$ and zeta potential $-42.9 \mathrm{mV}$ (Fig. 2a) and the average mean size of PVP doped AgNPs was $54.09 \mathrm{~nm}$ with 0.64 PDI and zeta potential $-16.9 \mathrm{mV}$ (Fig. 2b).

\section{Nanoparticle tracking analysis (NTA)}

Nanoparticle tracking analysis enables sample visualization and provides approximate particle dispersion which is very useful features. The images of NTA are shown in Fig. 3. From the NTA data it is clear that the aggregation between the particles is very less and particles are uniformly dispersed. 
Fig. 3 NTA images of TSCAgNPs a and PVP-AgNPs b. NTA images are clearly showing the uniform dispersion of prepared silver nanoparticles
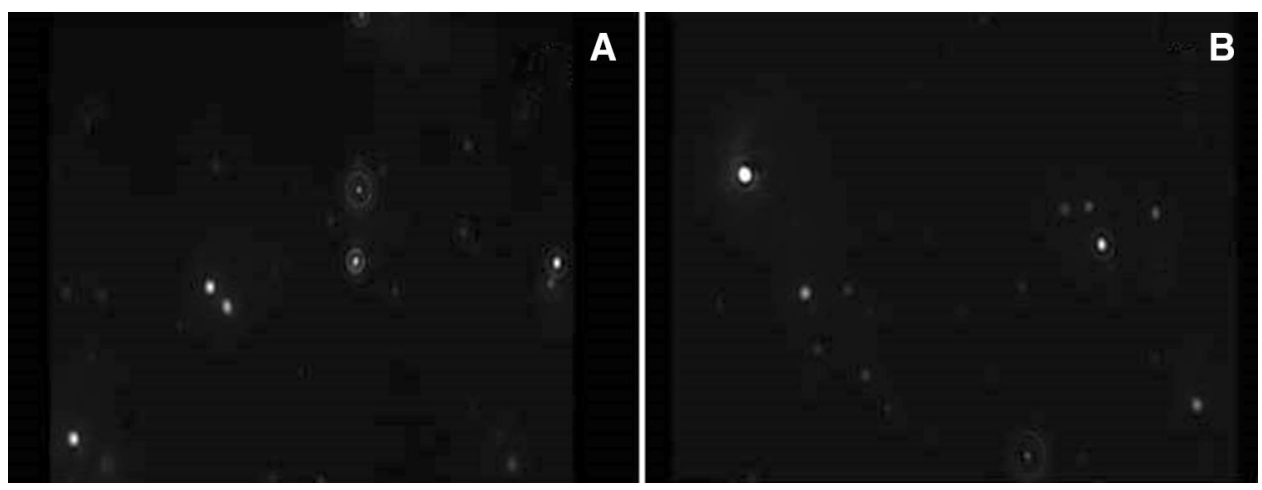

Fig. 4 SEM images of TSCAgNPs a and PVP-AgNPs b. SEM images are clearly showing the size $\sim 100 \mathrm{~nm}$ and spherical shape of the prepared silver nanoparticles
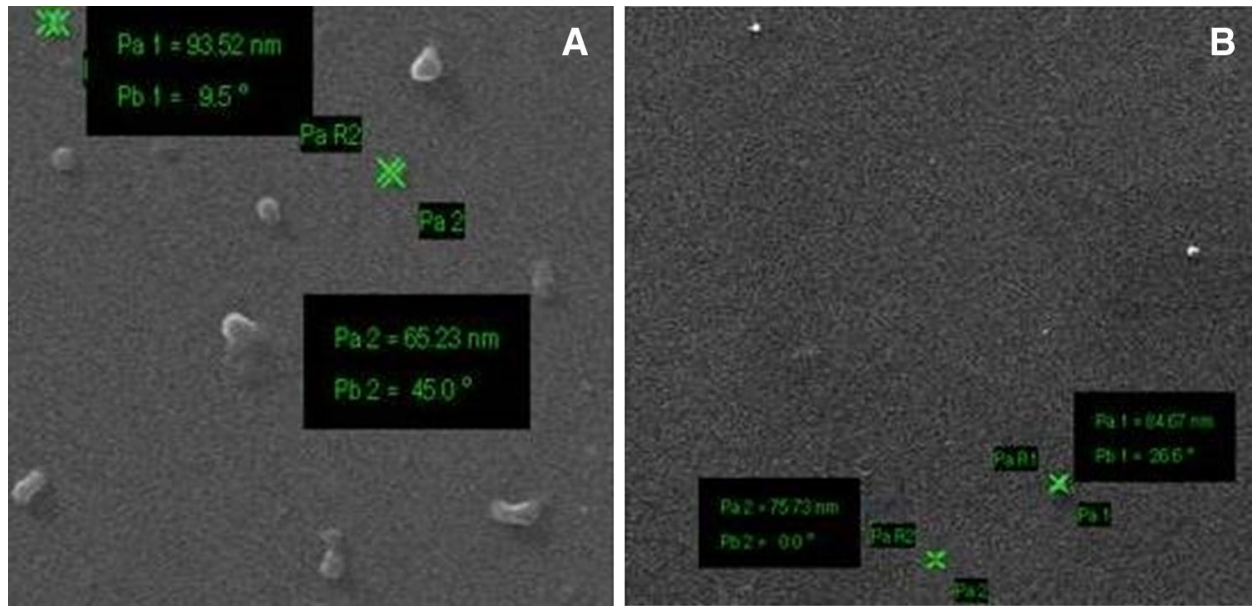

\section{Scanning electron microscopy (SEM)}

Scanning electron microscopy was done to confirm the size and shape of silver nanoparticles. From the SEM images shown in Fig. 4 it is clear that the particles are spherical in shape and are below $100 \mathrm{~nm}$ in size. The PVP-AgNps are slightly larger in size as compared to TSC-AgNPs.

\section{Antibacterial efficacy}

\section{Disc diffusion assay}

After $24 \mathrm{~h}$ of incubation the zones of growth inhibition around each of the disc were measured to the nearest millimeter. Highest ZoI (Table 1) was observed in TSCAgNPs followed by PVP doped AgNPs (Fig. 5). The diameter of the zone is related to the susceptibility of the isolate and to the diffusion rate of the TSC-AgNPs through the agar medium. Hence, the clear zone of inhibition around the TSC-AgNPs indicates its higher bactericidal activity than PVP-AgNPs.

Absorbance value was taken at regular interval at $600 \mathrm{~nm}$ and a graph was plotted to examine the growth inhibition by citrate and PVP coated AgNPs. TSC coated
Table 1 Antibacterial activity of silver nanoparticles on E. coli and S. aureus

\begin{tabular}{lrr}
\hline Sample & \multicolumn{2}{c}{ Zone of inhibition $(\mathrm{mm})$} \\
\cline { 2 - 3 } & \multicolumn{1}{c}{ E. coli } & \multicolumn{1}{c}{ S. aureus } \\
\hline $\mathrm{TSC}^{-\mathrm{AgNO}_{3}}$ & $2 \pm 0.34$ & $3.5 \pm 0.05$ \\
$\mathrm{PVP} \mathrm{AgNO}_{3}$ & $0.5 \pm 0.12$ & $2.5 \pm 0.16$ \\
Gentamycin $(1 \mathrm{mg} / \mathrm{ml})$ & $5 \pm 0.22$ & $6 \pm 0.26$ \\
\hline
\end{tabular}

AgNPs showed more bacterial growth inhibition than PVPAgNPs, which is exhibiting slight inhibition of bacterial growth than control culture.

\section{Determination of MIC and MBC}

Absorbance value was taken at regular intervals at $600 \mathrm{~nm}$ and a graph was plotted in the origin to examine the growth inhibition by TSC-AgNPs (Fig. 6a) and PVP-AgNPs (Fig. 6b).

MIC value (Table 2) for TSC-AgNPs is $26.75 \mu \mathrm{g} / \mathrm{ml}$ and MBC (Table 2) value is $53 \mu \mathrm{g} / \mathrm{ml}$. In case of PVPAgNPs MIC value is $269.5 \mu \mathrm{g} / \mathrm{ml}$. 

silver nanoparticles against a Gram-negative E. coli and b Gram-positive $S$. aureus, (-) negative control; distilled water, Gentamycin
Fig. 5 Antimicrobial activity of $(+)$ positive control;
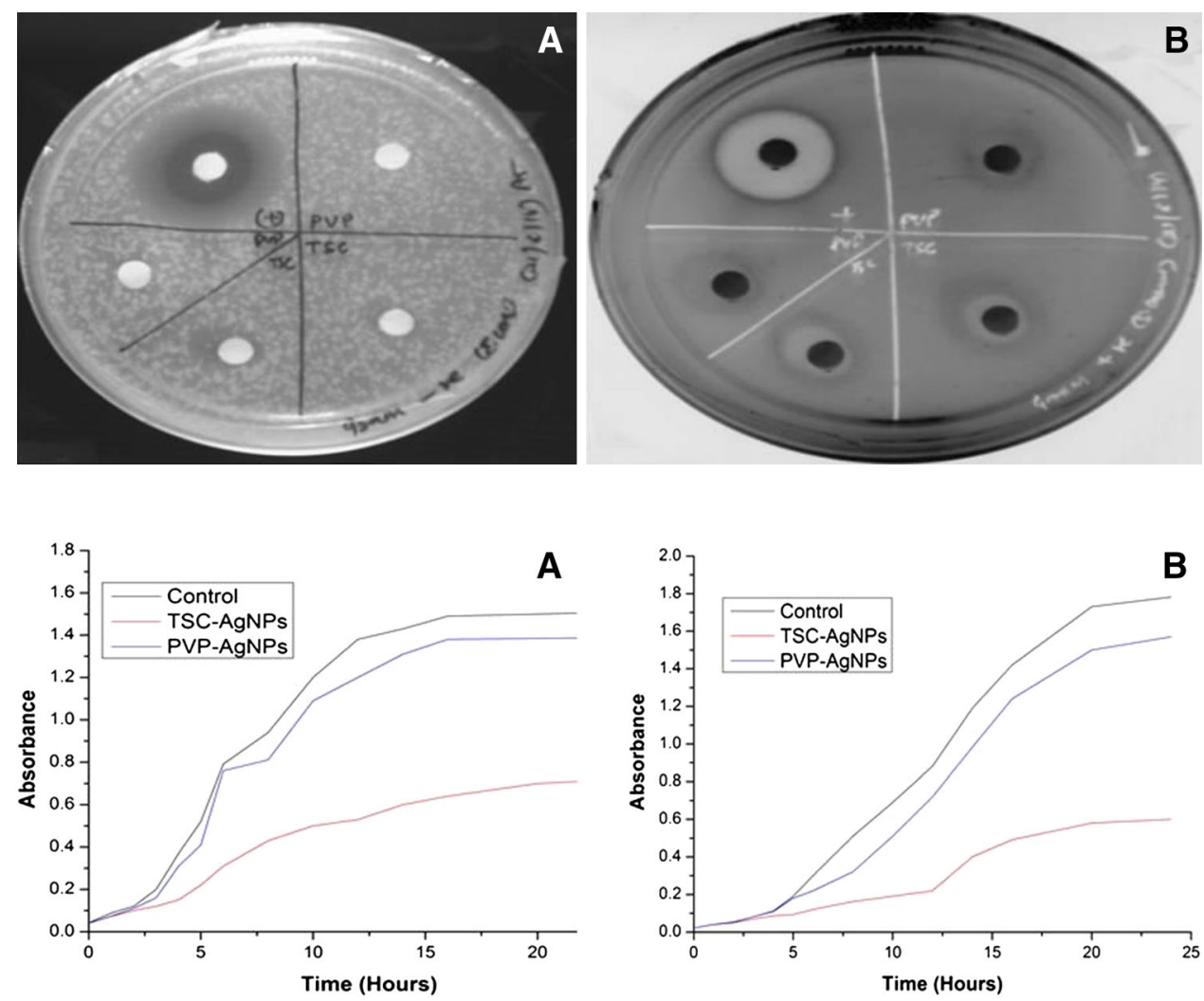

Fig. 6 Effect of different AgNPs on a $S$. aureus and b E. coli growth

Table 2 Determination of MIC and $\mathrm{MBC}$ of silver nanoparticles on E. coli and S. aureus

\begin{tabular}{|c|c|c|c|c|}
\hline \multirow[t]{2}{*}{ Sample } & \multicolumn{2}{|l|}{ E. coli } & \multicolumn{2}{|l|}{ S. aureus } \\
\hline & $\mathrm{MIC}(\mu \mathrm{g} / \mathrm{ml})$ & $\mathrm{MBC}(\mu \mathrm{g} / \mathrm{ml})$ & $\operatorname{MIC}(\mu \mathrm{g} / \mathrm{ml})$ & $\mathrm{MBC}(\mu \mathrm{g} / \mathrm{ml})$ \\
\hline TSC-AgNO 3 & 26.75 & 53 & 13.48 & 26.75 \\
\hline PVP-AgNO 3 & 269.5 & $\leq 269.5$ & 67.41 & 134.83 \\
\hline
\end{tabular}

\section{DNA fragmentation assay}

The gel clearly indicated the typical ladder in TSC-AgNPs (Fig. 7). DNA damage was more visible in TSC-AgNPs whereas no fragmentation was observed in PVP doped AgNPs.

\section{Discussion}

With the increase in mutations of microbial organisms, developing resistance to multiple antibiotics, and the continuing emphasis on health-care costs, many scientists researchers are trying to develop novel, cost effective antimicrobial agents. Such problems and needs have led to the resurgence of finding a broad spectrum antiseptic activity better than antibiotics [22].

The nanoparticles were prepared by modifying a routinely used method involving borohydride reduction of silver nitrate in the presence of stabilizing agents. TSC-
AgNPs (34 nm) and PVP-AgNPs (54 nm) were synthesized using different reducing and stabilizing agents, also the zeta potential of both the nanoparticles was found to be -42.9 and $-16.9 \mathrm{mV}$, respectively. The decrease in zeta potential from -42.9 to $-16.9 \mathrm{mV}$ in PVP-AgNPs is due to the charge neutralization by capping of the silver nanoparticles with PVP. Silver collide was directly used to test the antibacterial activity so the concentration mentioned in the entire result was determined theoretically. One of the important criterions for nanoparticle production is the prevention of particle aggregation during synthesis. The nanoparticles can be stabilized either by stearic or electrostatic forces. The steric stabilization can be achieved by doping polymers such as PVP, whereas the electrostatic stabilization can be attained by surface modifiers such as sodium borohydride and sodium citrate. AgNPs synthesized using TSC as a reducing and stabilizing agent showed higher antibacterial activity as compared to PVP-AgNPs. From this result we can conclude that electrostatic stabilization was more effective than steric stabilization. 
Fig. 7 DNA fragmentation assay, a Lane $M 100 \mathrm{bp}-3 \mathrm{Kbp}$ ladder, Lane 1 untreated E. coli culture, Lane 2 E. coli treated with PVP-AgNPs, Lane 3 E. coli treated with TSCAgNPs; b Lane $M 250$ bp$25 \mathrm{Kbp}$ ladder, Lane 1 untreated $S$. aureus culture, Lane $2 S$. aureus treated with PVP-

AgNPs, Lane 3 S. aureus treated with TSC-AgNPs

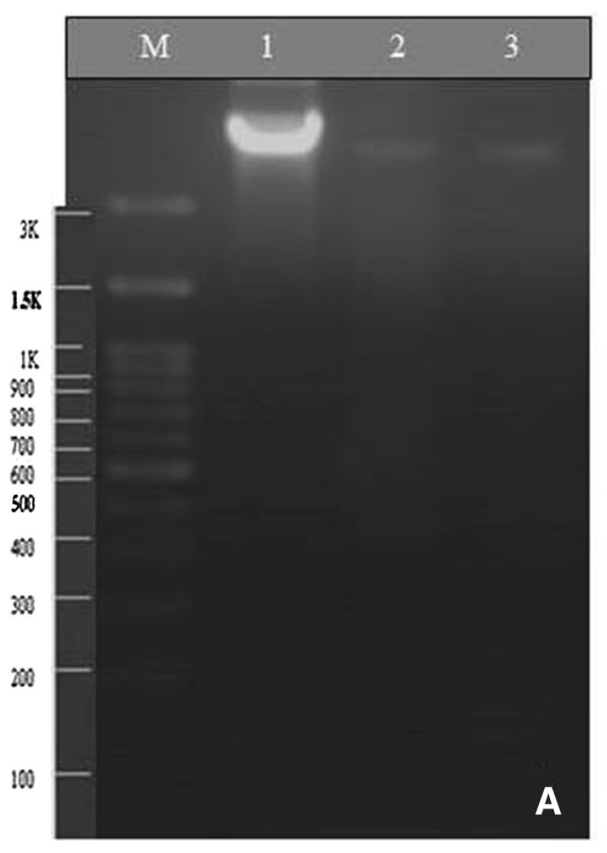

Further, the capping of the silver nanoparticles with PVP resulted in the mild increase in the particle size of the silver nanoparticles.

Nanoparticle tracking analysis, which was first commercialized in 2006, is an innovative system for sizing particles from about 30 to $1000 \mathrm{~nm}$, with the lower detection limit being dependent on the refractive index of the nanoparticles. This technique combines laser light scattering microscopy with a charge-coupled device (CCD) camera, which enables the visualization and recording of nanoparticles in solution. The NTA software is then able to identify and track individual nanoparticles moving under Brownian motion and relates the movement to a particle size according to the following formula derived from the Stokes-Einstein equation. One of the main constraints of DLS is the impact that few large particles, such as dust, may have on the average/mean size. The NTA technique is based on the tracking of single particles, whereas DLS measures a bulk of particles with a strong bias to the largest particles present in the sample. Therefore, the performance of NTA is expected to be less sensitive than DLS to the presence of minute amounts of large particles. The influence of large particles on NTA and DLS results has been summarized and reported earlier [18]. Furthermore, the SEM results also confirm the size and spherical shape of the nanoparticles.

Due to the smaller size of the AgNPs the surface area was quite larger, hence the interaction with the bacterial cell was probably more than PVP-AgNPs. In the recent decades, nanoparticles have received considerable attention, largely due to their broad range of bioactivities and distinctive mode of action [23].

Gel electrophoresis result also indicated that TSCAgNPs caused significant DNA fragmentation. The cause of DNA damage can be explained by the mechanism of the bactericidal effect of AgNPs. The reason for this size dependency is probably a combination of the particles' ability to react with and collectively penetrate the cell membrane by the cumulative higher surface to volume ratio of nanoparticles. It is well documented that Grampositive and Gram-negative bacteria have differences in their membrane structure as the peptidoglycan layer is thicker and contains Teichoic acid [23]. Therefore, the efficacy of the Ag nanoparticles against $S$. aureus may be due the difference in the membrane structure. To confirm this hypothesis, further comparative study between various gram-negative and gram-positive bacterial species was done. Since the peptidoglycan layer is a specific feature of bacteria and is not applicable to mammalian cells, then the antibacterial effect of Ag nanoparticles must be associated with the peptidoglycan layer. Therefore, the applications of $\mathrm{Ag}$ nanoparticles as an antibacterial agent will be easier and more specific to use as a textile coating, or coatings of surgical instruments etc.

It is known that small (less than $5 \mathrm{~nm}$ ) metallic particles show electronic effects that are defined as changes in the local electronic structure of the surface. These effects enhance the reactivity of the nanoparticle surfaces. 


\section{Conclusion}

The size of TSC-AgNPs was $34 \mathrm{~nm}$ and PVP-AgNO 3 was $54 \mathrm{~nm}$. Single SPR peak confirmed no aggregation occurred in both the synthesis process. The present study also concluded that electrostatic stabilization was more effective than steric stabilization, hence citrate coated AgNPs were smaller in size and have shown less polydispersity. Moreover, AgNPs have also shown antibacterial activity against E. coli and $S$. aureus as observed by the disc diffusion assay and growth kinetics curve. Furthermore significant DNA damage was possibly be due to interaction of silver ions with the DNA of microorganism that leads to the instability of DNA and thereby, interfering with its ability to replicate. However, the exact molecular mechanism of targeting is not fully understood; hence it warrants further investigation into the molecular mechanism.

Acknowledgments Arindam Dey and Abhirup Dasgupta are thankful to Nano Biotech Lab for their summer training. Vijay Kumar and Aakriti Tyagi are thankful to DBT and UGC for their fellowship respectively.

Open Access This article is distributed under the terms of the Creative Commons Attribution 4.0 International License (http://crea tivecommons.org/licenses/by/4.0/), which permits unrestricted use, distribution, and reproduction in any medium, provided you give appropriate credit to the original author(s) and the source, provide a link to the Creative Commons license, and indicate if changes were made.

\section{References}

1. De, M., Ghosh, P.S., Rotello, V.M.: Applications of nanoparticles in biology. Adv. Mater. 20, 4225 (2008)

2. Lu, A.H., Salabas, E.L., Schüth, F.: Magnetic nanoparticles: synthesis, protection, functionalization, and application. Angew. Chem. Int. Ed. 46, 1222-1244 (2007)

3. Chaudhuri, R.G., Paria, S.: Core/shell nanoparticles: classes, properties, synthesis mechanisms, characterization, and applications. Chem. Rev. 11 112(4), 2373-2433 (2012)

4. Sharma, V.K., Yngard, R.A., Lin, Y.: Silver nanoparticles: green synthesis and their antimicrobial activities. Adv. Colloid Sur. Interface 145, 83 (2009)

5. Krutyakov, Y.A., Kudrinskiy, A.A., Olenin, A.Y., Lisichkin, G.V.: Synthesis and properties of silver nanoparticles: advances and prospects. Russ. Chem. Rev. 77(3), 233-257 (2008)

6. Monteiroa, D.R., Gorupb, L.F., Takamiyaa, A.S., Ruvollo-Filhob, A.C., de Camargob, E.R., Barbosa, D.B.: The growing importance of materials that prevent microbial adhesion: antimicrobial effect of medical devices containing silver. Int. J. Antimicrob. Agents 34, 103-110 (2009)

7. García-Barrasa, J., López-de-Luzuriaga, J.M., Monge, M.: Silver nanoparticles: synthesis through chemical methods in solution and biomedical applications. Cent. Eur. J. Chem. 9(1), 7-19 (2011)
8. Fabrega, J., Luoma, S.N., Tyler, C.R., Galloway, T.S., Lead, J.R.: Silver nanoparticles: behaviour and effects in the aquatic environment. Environ. Int. 37, 517 (2011)

9. Dallas, P., Sharma, V.K., Zboril, R.: Silver polymeric nanocomposites as advanced antimicrobial agents: classification, synthetic paths, applications, and perspectives. Adv. Colloid Interface Sci. 166, 119 (2011)

10. Kim, K.J., Sung, W.S., Moon, S.K., Choi, J.S., Kim, J.G., Lee, D.G.: Antifungal effect of silver nanoparticles on dermatophytes. J. Microbiol. Biotechnol. 18, 1482-1484 (2008)

11. Nadworny, P.L., Wang, J., Tredget, E.E., Burrell, R.E.: Antiinflammatory activity of nanocrystalline silver in a porcine contact dermatitis model. Nanomed. Nanotechnol. Biol. Med. 4(3), 241-251 (2008)

12. Rogers, J.V., Parkinson, C.V., Choi, Y.W., Speshock, J.L., Hussain, S.M.: A Preliminary assessment of silver nanoparticle inhibition of monkeypox virus plaque formation. Nanoscale Res. Lett. 3, 129-133 (2008)

13. Emily, A., Morell, B.A., Balkin, D.M.: Methicillin-resistant Staphylococcus aureus: a pervasive pathogen highlights the need for new antimicrobial development. Yale J. Biol. Med. 84(4), 223-233 (2010)

14. Meng, J., Zhao, S., Doyle, M.P., Joseph, S.W.: Antibiotic resistance of Escherichia coli O157:H7 and O157:NM isolated from animals, food, and humans. J. Food Prot. 61(11), 1511-1514 (1998)

15. Kim, J.S., Kuk, E., Yu, K.N., Kim, J.H., Park, S.J., Lee, H.J., Kim, S.H., Park, Y.K., Park, Y.H., Hwang, C.Y., Kim, Y.K., Lee, Y.S., Jeong, D.H., Cho, M.H.: Antimicrobial effects of silver nanoparticles. Nanomed. Nanotechnol. Biol. Med. 3(1), 95-101 (2007)

16. Fang, J., Zhong, C., Mu, R.: The study of deposited silver particulate films by simple method for efficient SERS. Chem. Phys. Lett. 401, 271-275 (2005)

17. Verma, A.K., Sachin, K., Saxena, A., Bohidar, H.B.: Release kinetics from bio-polymeric nanoparticles encapsulating protein synthesis inhibitor-cycloheximide, for possible therapeutic applications. Curr Pharm Biotechnol 6(2), 121-130 (2005)

18. Filipe, V., Hawe, A., Jiskoot, W.: Critical evaluation of nanoparticle tracking analysis (NTA) by nanosight for the measurement of nanoparticles and protein aggregates. Pharm. Res. 27(5), 36 (2010)

19. Fatma, S., Talegaonkar, S., Iqbal, Z., Panda, A.K., Negi, L.M., Goswami D.G., Tariq M.: Novel flavonoid-based biodegradable nanoparticles for effective oral delivery of etoposide by P-glycoprotein modulation: an in vitro, ex vivo and in vivo investigations. Drug Deliv. 1-12 (2014)

20. Ruparelia, J.P., Chatterjee, A.K., Duttagupta, S.P., Mukherji, S.: Strain specificity in antimicrobial activity of silver and copper nanoparticles. Acta Biomater. 4, 707-716 (2008)

21. Tyagi, A., Agarwal, S., Leekha, A., Verma, A.K.: Effect of mass and aspect heterogeneity of chitosan nanoparticles on bactericidal activity. Int. J. Adv. Res. 2(8), 357-367 (2014)

22. Jones, S.A., Bowler, P.G., Walker, M., Parsons, D.: Controlling wound bioburden with a novel silver-containing Hydrofiber ${ }^{\mathbb{B}-}$ dressing. Wound Repair Regen. 12(3), 288-294 (2004)

23. Jena, P., Mohanty, S., Mallick, R., Jacob, B., Sonawane, A.: Toxicity and antibacterial assessment of chitosancoated silver nanoparticles on human pathogens and macrophage cells. Int. J. Nanomed. 7, 1805-1818 (2012) 